\title{
CARACTERIZACIÓN EXOMORFOLÓgICA Y MICROGRÁFICA DE CHEILANTHES PRUINATA, ESPECIE TÓXICA PARA EL GANADO Y MEDICINAL EN HUMANOS EN LA REGIÓN ANDINA
}

\author{
ESTELA N. FLORES ${ }^{1}$, LAURA M. CALIFANO² ${ }^{2}$ ALBERTO A. GURNI ${ }^{1}$ y NILDA D. VIGNALE${ }^{1}$
}

\begin{abstract}
Resumen: El objetivo del trabajo fue definir, mediante la aplicación del método micrográfico, los caracteres anatómicos de valor diagnóstico requeridos para identificar la parte aérea de la especie denominada comúnmente "doradilla" (Cheilanthes pruinata Kaulf.) causal de intoxicaciones en el ganado menor en el NO de Argentina y que también es utilizada en medicina herbolaria en los Andes de Perú y Bolivia como analgésico y antihemorrágico. Se aplicó la técnica de disociado leve a hojas, órganos presumiblemente tóxicos y/o medicinales. Los resultados indican que, en láminas foliares, los pelos glandulares de pie uni y bicelulares, y cabeza secretora unicelular, las esporas triletes y los leptosporangios con anillo de dehiscencia vertical son las referencias válidas y en raquis y pecíolos los pelos tectores bicelulares de célula basal recta o acodada y célula apical aguzada fácilmente separable y fibro y macroesclereidas. Esta información es útil para reconocer la especie, tanto en contenido ruminal en caso de sospecha de intoxicación, como para realizar controles de calidad botánicos en muestras de herboristería.
\end{abstract}

Palabras clave: Doradilla, Cheilanthes pruinata, intoxicación, medicina herbolaria, micrografía.

\begin{abstract}
Summary: Exomorphologic and micrographic characterization of Cheilanthes pruinata, a toxic species for livestock and medicinal for humans in the Andean region. Exomorfologic and micrographic characterization of "doradilla", toxic specie to livestock and also medicinal for humans in Andean region. The study aimed to define, through the application of the micrographic method, those anatomical characters of diagnostic value required to identify the aerial parts of the specie commonly referred as "doradilla" (Cheilanthes pruinata Kaulf.) cause of poisoning in small livestock in the NW of Argentina, and which is also used in herbal medicine as analgesic and anti-haemorrhagic in the Andes of Bolivia and Peru. Mild dissociated technique was applied to leaves, presumably toxics and/or medicinal organs. Results show that, in leaf blades, glandular hairs with one- and two-cell stalk and single-cell secretory head as well as the trilete spores and leptosporangia with vertical ring dehiscence are valid references; and in rachis and petioles, bicellular protector hairs with basal straight or angled cell and easily separable sharp apical cell, along with fibro and macroesclereidas. Such information is useful to recognize the species both in ruminal contents in case of suspicion of intoxication as for quality control of botanical herbal samples.
\end{abstract}

Key words: Doradilla, Cheilanthes pruinata, intoxication, herbal medicine, micrographic method.

\section{INTRODUCCIÓN}

Trabajos etnobotánicos han registrado la toxicidad para el ganado de "doradilla" o "chujcho", Cheilanthes pruinata Kaulf. (Pteridaceae) (Fig. 1)

\footnotetext{
1 Laboratorio de Botánica Sistemática y Etnobotánica, Cátedra de Botánica Sistemática y Fitogeografía, Facultad de Ciencias Agrarias, Universidad Nacional de Jujuy. Alberdi 47 (4600) S. S. de Jujuy. Argentina, ndvignale@yahoo.com.ar.

2 INTA, Estación Experimental Agropecuaria Abra Pampa. Maestro Daniel Domínguez 17 (4630) Humahuaca. Jujuy. Argentina,califano.laura@inta.gob.ar.
}

en la cuenca del río Iruya (Prov. Salta, Argentina). Se trata de una especie que afecta el sistema nervioso, especialmente en crías de ovinos y caprinos de no más de un año de edad, produciendo la intoxicación luego de 48 a 72 horas del consumo. En los afectados progresivamente ocasiona depresión, anorexia, fibrilaciones musculares en miembros y ataxia locomotriz, posición decúbito y, finalmente, el deceso. Los animales la consumen cuando se encuentra seca en primavera (septiembrediciembre) inducidos por la escasez de forraje, en las ocasiones en que son trasladados a puestos de altura (Califano \& Echazú, 2011; 2013). 


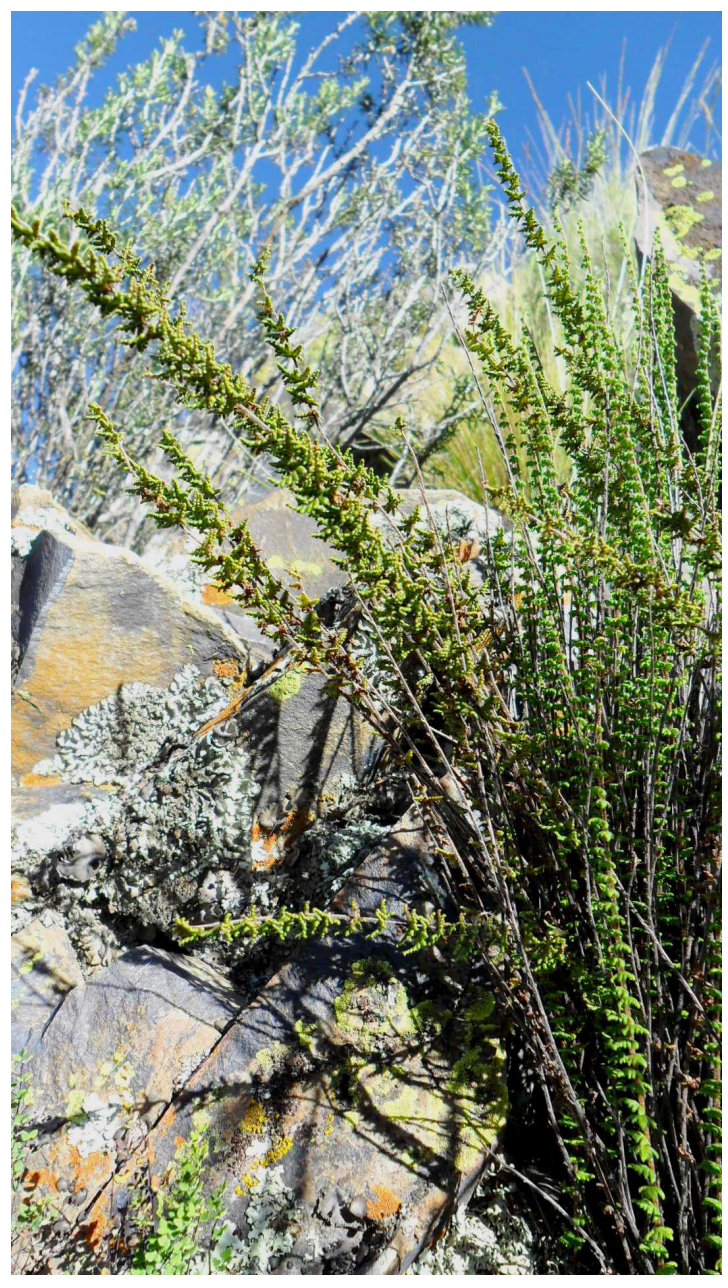

Fig. 1. C. pruinata en su ambiente natural.

Esta especie se halla desde Perú hasta el norte de Chile y en el NO y centro de Argentina, alcanzando los 4800 m s.n.m. De hábitos saxícolas, es frecuente en fisuras de rocas y sitios sombríos, siendo uno de los helechos que crece a mayor altura en Argentina (De la Sota et al., 2001). Es xerófila, presenta rizomas rastreros con escamas castaño-oscuras y frondes de 15-30 cm de largo; éstas últimas se caracterizan por presentar: -1- pecíolos robustos, castaño-oscuros, con escasos pelos y escamas, -2- láminas bipinnadapinnatífidas, de contorno linear, raquis piloso, pinnas alternas, deltoides, deciduas o pubescentes, con márgenes reflexos continuos, lobados o crispados (De la Sota, 1977; Rodríguez, 1995; Arteta, 2008). Según Morbelli \& Michelena (1989) las esporas de Cheilanthes son subtriangulares a globosas en vista polar. Hernández et al. (2008) describen en rizomas y raíz de C. pruinata la presencia de braquiesclereidas y macroesclereidas con las paredes extremadamente engrosadas.

En gran parte del NO de Argentina el conocimiento y la habilidad de utilizar vegetales para curar afecciones es notable, en parte por la influencia de los curanderos o médicos itinerantes andinos denominados "kallawayas". Por ello la "doradilla" o "chujcho" ha sido registrada como medicinal en abundante bibliografía referida al área andina. Se citan usos para afecciones como dolor al orinar, dolor abdominal postparto, inducción del parto (acelerar partos distócicos o inducción de abortos), para disminuir la hemorragia del parto, dolor estomacal, dolor menstrual, cefaleas, diurético, para la tos, el mal de Chagas y como abortivo (Girault, 1987; Roersch, 1994; Pestalozzi Schmid, 1998; Vandebroek \& Thomas, 2003; Villagrán \& Castro, 2003; Navarrete et al., 2006; Thomas et al., 2008).

Si bien la composición química de esta especie no ha sido estudiada, la descripción de los signos de intoxicación que provoca en el ganado muestran que se trataría de un depresor del sistema nervioso y consecuentemente produciría una disminución en la percepción del dolor; estos efectos se encuentran relacionados con los usos medicinales descriptos, sobre todo cuando es utilizada como analgésica.

Actualmente existe una tendencia generalizada del consumo de productos biológicos naturales, la que, en parte, se sustenta en el supuesto de la inocuidad de las plantas y de su superioridad sobre las drogas sintéticas. Esto motiva la elaboración y comercialización de gran cantidad de productos vegetales de consumo masivo, y contribuye a la circulación y uso de hierbas medicinales sin un conocimiento profundo y desprovisto de estricto control comercial. Este panorama implica un peligro para la salud de los miembros de la sociedad que los consumen (Pochettino et al., 2008).

Siguiendo esta tendencia, en Argentina se comercializa bajo el nombre de "doradilla" o "torarilla", Anemia australis (Mickel) M. Kessler \& A.R. Sm. (Luján et al., 2000) y podría estar también comercializándose $C$. pruinata, tal como se menciona en fuentes bibliográficas de Perú y Bolivia (Girault, 1987,; Pestalozzi Schmid, 1998; Vandebroek \& Thomas, 2003; Villagrán \& Castro, 2003; Navarrete et al., 2006; Thomas et al., 2008).

La información sobre el uso terapéutico de las 


\section{E. N. Flores et al. - Caracterización exomorfológica y micrográfica de Cheilanthes pruinata}

plantas medicinales se basa principalmente en conocimientos empíricos que pueden haber sido tomados de fuentes etnobotánicas; pero debido a que todavía se desconocen las características anatómicas de muchas especies de la flora presuntamente medicinal, dichas plantas son difíciles de identificar cuando se hallan en su fase vegetativa o cuando el material está muy picado o pulverizado para su comercialización (Luján et al., 2000).

Por otro lado, las intoxicaciones del ganado por plantas tóxicas son frecuentes en Argentina y su diagnóstico usualmente es complicado. Tanto los signos, como las lesiones macroscópicas y microscópicas no son claras, lo que constituye un desafío para los veterinarios (Zeinsteger et al., 2011). Las plantas tóxicas requieren disponer de la posibilidad de ser identificadas a partir de caracteres micrográficos. En efecto, es común que a la hora del diagnóstico el único material disponible es el contenido ruminal, constituido por fragmentos de los órganos de las plantas causantes de la intoxicación. En este tipo de muestras generalmente no se aprecian detalles exomorfológicos que permitan su determinación taxonómica. Al respecto merecen mención los trabajos de Zeinsteger (2010) y Zeinsteger et al. $(2004,2011)$ sobre micrografía de plantas tóxicas del NE de Argentina que brindan herramientas para un diagnóstico diferencial a partir de fragmentos de vegetales hallados en el rumen. Entre las especies tóxicas del NE que han sido abordadas desde esta perspectiva se pueden mencionar Baccharis coridifolia DC y Pascalia glauca Ortega. Sin embargo, existe un vacío de información para las especies del NO, constituyéndose este trabajo en uno de los primeros aportes, cuyo objetivo es definir, mediante la aplicación del método micrográfico, los caracteres citológicos e histológicos de valor diagnóstico requeridos para identificar la parte aérea de $C$. pruinata en materiales trozados o fragmentados, en los cuales los caracteres exomorfológicos no resultan perceptibles. De este modo se propone aportar, a través de una herramienta de fácil y de rápida aplicación en laboratorios, información necesaria para garantizar la calidad botánica de las muestras comercializadas como así también útil para la determinación del vegetal causante de intoxicaciones en el ganado (cuando lo que se dispone son fragmentos presentes en el contenido ruminal).

\section{Materiales y Métodos}

Se recolectaron muestras en el área comprendida por los departamentos Iruya (Salta) y Humahuaca (Jujuy), donde se registran casos de intoxicación en ganado con esta especie. El material vegetal herborizado y estudiado se encuentra depositado en el Herbario del Museo de Ciencias Naturales de la Facultad de Ciencias Naturales (Universidad Nacional de Salta) cuya sigla, según Index Herbariorum (Holmgren et al., 1990) es MCNS.

Su identificación se realizó mediante el análisis de las características exomorfológicas, con las claves dicotómicas disponibles en bibliografía especializada (De la Sota, 1977; De la Sota et al., 2001).

\section{Material estudiado}

Cheilanthes pruinata Kauf.

ARGENTINA. Salta, Santa Victoria, (Poscaya) Nazareno, 3210 m s.m., 06-II-2011, Califano \& Echazú 168 (MCNS).

ARGENTINA. Salta, Iruya, camino a Rodeo Colorado entre Vizcarra y Campamento, 3864 m s.m., 06-VI-2012, Califano \& Echazú 186 (MCNS).

ARGENTINA. Jujuy, Humahuaca, Ruta Provincial $\mathrm{N}^{\circ} 73$ a la altura de la localidad de Pucara, 12-III-2012, Califano \& Echazú 185(MCNS).

ARGENTINA. Salta, Iruya, entre Miyuyoc y Abra del Cóndor, 21-I-2013, Califano \& Echazú 188 (MCNS).

\section{Métodos}

Del método micrográfico se aplicó la técnica de disociado leve a hojas. La misma consiste en el tratamiento de trozos de material con solución acuosa de $\mathrm{NaOH} 5 \%$, a ebullición, por espacio de 5 min., posterior lavado y colocación de una pequeña porción entre porta y cubreobjetos para su observación al microscopio óptico (D'Ambrogio de Argüeso, 1986; Gattuso \& Gattuso, 1999; Gurni, 2014).

Las observaciones se realizaron con Microscopio Trinocular Carl Zeiss, modelo Axiostar Plus que lleva incorporada una cámara compacta digital Cannon modelo Powershot A640 con la que se tomaron la fotomicrografías. 


\section{Resultados y Discusión}

En láminas foliares se observaron células parenquimáticas y epidérmicas, pelos glandulares con pie alargado unicelular cuya longitud alcanza el doble de longitud de la cabeza (Fig. 2 A) y bicelular (Fig. 2 B-C), ambos con cabeza secretora unicelular, leptosporangios con anillo de dehiscencia lateral (Fig. 2 D) conteniendo las esporas triletes, las que se aprecian según visión distal, lateral y proximal (Figs. 2 E-F).

En raquis y pecíolos foliares se observó parénquima, pelos tectores bicelulares cuya célula apical de extremo aguzado se separa con facilidad de la célula basal, recta o generalmente acodada en la base, quedando ésta última solitaria y con su extremo romo en la mayoría de las observaciones (Fig. $2 \mathrm{G}$ ), fragmentos de tejidos de conducción y esclereidas de dos tipos: largas y angostas, de extremos aguzados (fibroesclereidas) y otras anchas y más cortas, de extremos romos (macroesclereidas), todas con paredes muy engrosadas (Figs. $2 \mathrm{H}-\mathrm{I}$ ).

La mayoría de las especies medicinales y tóxicas estudiadas aplicando el método micrográfico pertenecen a familias botánicas de la División Magnoliophyta (Angiospermae); un número reducido de especies pertenecen al grupo de los Helechos, como la que se aborda en el presente análisis. Independientemente de su ubicación taxonómica, el tejido epidérmico es el que revela

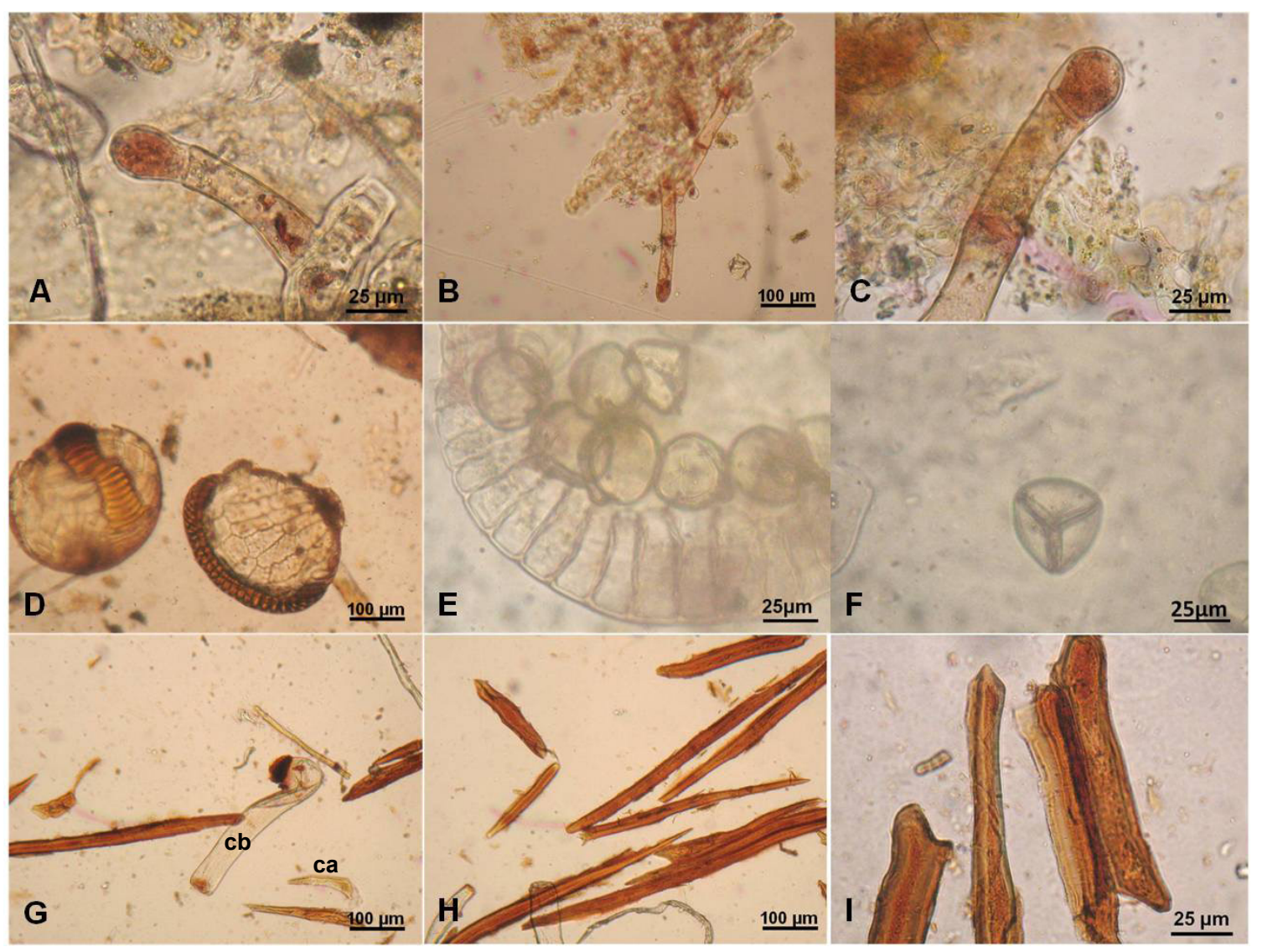

Fig. 2. Caracteres micrográficos de valor diagnóstico. A-F: Lámina foliar. A: Pelo glandular de pie y cabeza secretora unicelulares. B-C: Pelo glandular de pie bicelular y cabeza secretora unicelular. D: Leptoesporangios con anillo de dehiscencia vertical. E: Fragmento de leptoesporangio con esporas triletes. F: Espora trilete, vista proximal. G-I: Raquis y pecíolo foliar. G: Pelo tector bicelular, célula basal recta o acodada y apical de extremo aguzado. H: Fibroesclereidas. I: Macroesclereidas.

Referencias: ca: célula apical, cb: célula basal. 


\section{E. N. Flores et al. - Caracterización exomorfológica y micrográfica de Cheilanthes pruinata}

características diferenciales; en este caso se trata de la presencia de tricomas glandulares relativamente simples en su estructura, ya que están integrados por un pie bicelular o unicelular de notoria longitud (que duplica la longitud de la cabeza) y una cabeza secretora unicelular ovoide.

A nivel de láminas foliares son las estructuras reproductoras - leptoesporangios - las que aportan elementos identificatorios como la posición del anillo de dehiscencia, la pared de la cápsula y las esporas triletes. En coincidencia con la descripción de Morbelli \& Michelena (1989) las esporas, según vista polar, presentan forma subtriangular a globosa.

A nivel de raquis y pecíolos foliares es el tejido esclerenquimático el que ofrece aspectos diferenciales mediante las esclereidas con dos tipos morfológicos: alargadas y de extremos aguzados, típicas fibroesclereidas y anchas y más cortas, con extremos romos, macroesclereidas.

Ambos tipos de esclereidas coinciden con los citados, para rizomas y raíz de esta especie, por Hernández et al. (2008). En esta oportunidad se analizan los órganos de la especie que pueden ser tóxicos pues son consumidos por los animales; por tal motivo está ausente el estudio del rizoma.

Los elementos de valor diagnóstico aquí presentados se caracterizan por su capacidad de preservación en los distintos medios en los que pueden ser localizados; así por ejemplo en el rumen, instancia en la cual su detección cobra importancia en virtud de su valor como planta tóxica; asimismo expresan tolerancia a los procesos digestivos a los que son sometidos y se manifiestan tal los detalles presentados.

Cuando se necesita realizar la detección de la especie en material procedente de herboristerías en las que se encuentra fragmentado y hasta pulverizado, la visualización de los caracteres propuestos no presenta dificultades luego de practicadas las técnicas micrográficas aplicadas en este estudio ya que se trata de referencias citológicas que persisten al trozado y a la temperatura. Por ello son definidos estos identificadores micrográficos, porque pueden ser observados al microscopio fácilmente luego de provocar la desintegración de los tejidos mediante la disolución de la laminilla media, acción provocada por la aplicación de la técnica de disociado, llamada también disgregado, débil en el caso analizado.

\section{Conclusiones}

Los caracteres micrográficos de valor diagnóstico que posibilitarán certificar la presencia de hojas de esta especie en muestras trozadas en diferente grado, pulverizadas o tal como se presentan en contenido ruminal son los siguientes:

1. En láminas foliares estériles y fértiles: tricomas glandulares de pie uni y bicelular y cabeza secretora unicelular.

2. En láminas foliares fértiles: leptoesporangios con anillo de dehiscencia vertical y esporas triletes.

3. En raquis y pecíolos foliares: a. pelos tectores bicelulares con célula basal recta o acodada y célula apical de extremo aguzado, que se desprende con facilidad. b. fibro y macroesclereidas con paredes secundarias notoriamente engrosadas.

Los identificadores precedentemente señalados requieren su detección conjunta para certificar la presencia de hojas de la especie en las muestras problema, por lo menos los elementos citados en $1 \mathrm{y}$ 2. La visualización parcial de estos caracteres resulta insuficiente para cumplir con el propósito original de identificación específica.

Se trata del primer abordaje micrográfico de una especie de Cheilanthes con la finalidad de contribuir en su identificación en las situaciones particulares en las que es necesario recurrir a la consideración de datos endomorfológicos.

Esta opción de identificación micrográfica de material botánico, para la que se requiere disponer de laboratorio equipado con microscopio óptico, reducida cantidad de drogas y de elementos auxiliares y personal debidamente capacitado, podrá ser empleada para diagnósticos veterinarios diferenciales en caso de intoxicación, concretamente para conocer el origen taxonómico de la especie a partir de contenido ruminal como también para realizar controles de calidad botánicos en muestras de herboristería, cuando la especie se comercializa en base a sus propiedades medicinales, ya que el nombre vulgar "doradilla" es compartido con otras especies diferentes a la estudiada.

El estudio micrográfico constituye una estrategia auxiliar para la identificación botánica de material que permitirá arribar a un mejor diagnóstico clínico si se complementa con estudios hematológicos, hallazgos de necropsias e histopatología, brindando al veterinario información que permita determinar la especie responsable de la intoxicación. 
Los caracteres exomorfológicos de la especie a nivel de la lámina foliar, unidos a la detección de pelos glandulares y de las referencias reproductivas que evidencian los esporangios mediante la observación con lupa, pueden ser empleados para su identificación toda vez que su percepción resulte clara en el material problema. Cuando dicho material se presenta fragmentado se propone el empleo de los caracteres endomorfológicos propuestos y para ello las muestras deben ser sometidas a disociado leve y utilizar microscopio.

\section{Agradecimientos}

Al INTA EEA Abra Pampa por el apoyo brindado en la investigación y trabajos de campo. A la Secretaría de Ciencia y Técnica y Estudios Regionales (SeCTER) de la UNJu por el apoyo económico brindado al desarrollo de los Proyectos "Micrografía analítica comparativa de plantas alimenticias, medicinales y tóxicas de la subregión centro-oeste sudamericano", 08/A124 y "Plantas andinas de interés etnobotánico de la subregión centro-oeste sudamericano: caracterización micrográfica de órganos útiles y parámetros de calidad de semillas", 08/A175. A la AGENCIA NACIONAL DE PROMOCIÓN CIENTÍFICA Y TECNOLÓGICA por el financiamiento del Proyecto "La micrografía en la caracterización botánica de los cultivos andinos y su aporte al control de calidad de agroalimentos derivados", 30134 CA PICT que posibilitó lograr el equipamiento óptico necesario.

\section{Biblografía}

ARTETA, M. 2008. Etnobotánica de Plantas Vasculares en el centro poblado Llachón, Distrito Capachica, Departamento Puno. Tesis para optar al título profesional de Biólogo Facultad de Ciencias Biológicas y Agropecuarias, Universidad Nacional de San Agustín, Arequipa.

CALIFANO, L. M. \& F. ECHAZÚ. 2011. Especies vegetales tóxicas para el ganado en Humahuaca (Jujuy) e Iruya y Nazareno (Salta). Guía para $s u$ reconocimiento, la identificación y posibles tratamientos. INTA Centro Regional Salta-Jujuy.

CALIFANO, L. M. \& F. ECHAZÚ. 2013. Etnobotánica en comunidades pastoriles. Conocimiento tradicional sobre especies tóxicas para el ganado en la cuenca del río Iruya (Salta, Argentina). Bol. Soc. Argent. Bot. 48: 365-375.

D'AMBROGIO DE ARGÜESO, A. 1986. Manual de técnicas en histología vegetal. Editorial Hemisferio Sur. Buenos Aires. 83 pp.

DE LA SOTA, E. R. 1977. Pteridophyta. Parte II. En: CABRERA, A. L. (eds.), Flora de la Provincia de Jujuy. República Argentina. Col. Cient. INTA. Buenos Aires.

DE LA SOTA, E. R., O. G. MARTÍNEZ, M. PONCE , G. E. GIUDICE \& G. I. MICHELENA. 2001. Flora del valle de Lerma. Pteridaceae Rchb. 6. Herbario MCNS. Fac. Ciencias Naturales. UNSa, Salta.

GATTUSO, M. A. \& S. J. GATTUSO. 1999. Manual de procedimientos para el análisis de drogas en polvo. Universidad Nacional de Rosario. REUN. AUGM. UNESCO. RIPROFITO. Rosario.

GIRAULT, L. 1987. Curanderos itinerantes de los Andes. In: GIRAULT, L. (ed.), Kallawaya: Curanderos itinerantes de los andes. Quipus.

GURNI, A. A. 2014. Técnicas histológicas en investigación. En: ZARLAVSKY, G. E. (ed.). Histología vegetal. Técnicas simples y complejas. Sociedad Argentina de Botánica. Buenos Aires.

HERNÁNDEZ, M., P. ALBORNOZ, F. RODRÍGUEZ \& S. SERRANO. 2008. Anatomía de rizoma, raíz y micorrizas arbusculares en Cheilanthes pruinata Kaulf. y C. myriophylla Desv. (Pteridaceae) en el Noroeste Argentino. Lilloa 45: 73-82.

HOLMGREN, P. K., N. HOLMGREN \& C. BARNETT. 1990. Index Herbariorum. Part 1: The Herbaria of the World. 8th ed. New York Botanical Garden, Bronx.

LUJÁN, M. C., G. E. BARBOZA, S. WELER DE SERRA \& L. ARIZA ESPINAR. 2000. Control de calidad en dos helechos medicinales y su inserción en el mercado local. Stud. Bot. 19:75-94.

MORBELLI, M. A. \& I. G. MICHELENA. 1989. Palynological analysis of Cheilanthes Species (Adiantaceae-Pteridophyta) of Northwestern Argentina. Grana 28: 295-304.

NAVARRETE, H. B. L., J. GONZÁLEZ, D. AVILÉS, J. SALAZAR, F. MELLADO, J. ALBAN \& B. OLLGAARD. 2006. Helechos. Botánica Económica de los Andes Centrales. Universidad Mayor de San Andrés, La Paz.

PESTALOZZI SCHMID, H. U. 1998. Flora ilustrada alto andina. La relación entre hombre, planta $\mathrm{y}$ medio ambiente en el Ayllu Majasaya Mujlli. (Prov. Tapacarí, Dpto. Cochabamba, Bolivia Herbario Nacional de Bolivia y Herbario Nacional Forestal Martín Cárdenas, Cochabamba.

POCHETTINO, M. L., P. ARENAS, D. SÁNCHEZ \& R. CORREA. 2008. Conocimiento botánico tradicional, circulación comercial y consumo de 


\section{E. N. Flores et al. - Caracterización exomorfológica y micrográfica de Cheilanthes pruinata}

plantas medicinales en un área urbana de Argentina. Bol Latinoam Caribe Plant Med Aromat 7: 141-148.

RODRÍGUEZ, R. 1995. Pteridophyta. En: C. MARTICORENA \& R. RODRÍGUEZ (eds.), Flora de Chile. Universidad de Concepción.

ROERSCH, C. 1994. Plantas medicinales en el sur andino de Perú. Koeltz Scientific Books. Konigstein.

THOMAS, E., I. VANDEBROEK, P. GOETGHEBEUR, S. SANCA, S. ARRÁZOLA \& P. VAN DAMME. 2008. The relationship between plant use and plant diversity in the Bolivian Andes, with special reference to medicinal plant use. Hum. Ecol. 36: 861-879.

VANDEBROEK, I. \& E. THOMAS. 2003. Plantas medicinales para la atención primaria de la salud. El conocimiento de ocho médicos tradicionales de Apillampa (Bolivia). AMETRAC, Cochabamba.

VILlAGRÁN, C. \& V. CASTRO. 2003. Ciencia Indigena de Los Andes del Norte de Chile. Ed. Universitaria, Santiago.

ZEINSTEGER, P. A. 2010. Micrografía de plantas del nordeste argentino tóxicas para el ganado. Tesis Doctoral. Facultad de Farmacia y Bioquímica. Universidad de Buenos Aires.
ZEINSTEGER, P.A., A. GURNI \& A. PALACIOS. 2011. In vitro ruminal digestion and micrographic analysis of the poisonous plant Wedelia glauca (Ort.) Hoffm. Ex Hicken (Asteraceae). Dominguezia 27: 25-31.

ZEINSTEGER, P.A., G. KOZA, E. RÍOS, O. ACOSTA DE PÉREZ \& A. GURNI. 2004. Micrografía de Baccharis coridifolia DC (mio-mío) sometido a la acción in-vivo del licor ruminal. Estudio preliminar para el diagnóstico de la intoxicación. Anales de la XXV Sesión de Comunicaciones Cientificas, Facultad de Ciencias Veterinarias, UNNE, Corrientes. Diponible en: http://vet.unne. edu.ar/ComCientificas/sesion-04/Micrografia-deBaccharis.pdf. [Consultado en diciembre del 2014].

Recibido el 4 de febrero de 2015, aceptado el 14 de diciembre de 2015. 
